\title{
A Variational Finite Element Model for Large-Eddy Simulations of Turbulent Flows
}

\author{
Tomás Chacón Rebollo ${ }^{*}$ Roger Lewandowski ${ }^{\dagger}$
}

February 13, 2013

\begin{abstract}
We introduce a new Large Eddy Simulation model in a channel, based on the projection on finite element spaces as filtering operation in its variational form, for a given triangulation $\left\{\mathcal{T}_{h}\right\}_{h>0}$. The eddy viscosity is expressed in terms of the friction velocity in the boundary layer due to the wall, and is of a standard sub grid-model form outside the boundary layer. The mixing length scale is locally equal to the grid size. The computational domain is the channel without the linear sub-layer of the boundary layer. The no slip boundary condition (BC) is replaced by a Navier (BC) at the computational wall. Considering the steady state case, we show that the variational finite element model we have introduced, has a solution $\left(\mathbf{v}_{h}, p_{h}\right)_{h>0}$ that converges to a solution of the steady state Navier-Stokes Equation with Navier BC.
\end{abstract}

MCS Classification : 76D05, 76F65, 65M60,

\section{Introduction}

Numerical simulations of incompressible turbulent flows cannot be performed from the evolutionary Navier-Stokes Equations (NSE),

$$
\begin{aligned}
\partial_{t} \mathbf{v}+(\mathbf{v} \cdot \nabla) \mathbf{v}-\nu \Delta \mathbf{v}+\nabla p & =\mathbf{f}, \\
\nabla \cdot \mathbf{v} & =0,
\end{aligned}
$$

because of a great computational complexity due to the structure of the turbulence [30]. This is why various mathematical models derived from the NSE are used to simulate some features of turbulent flows, such as their statistical means or their large scales motions, this last way being known as "Large-Eddy Simulation" (LES), which is our concern in the present paper.

LES has attracted much attention these last two decades, especially because of the increasing of computational ressources, enabling to enlarge the range of scales that LES models might simulate. Basically, LES aims at computing filtered fields such as $\overline{\mathbf{v}}=G \star \mathbf{v}, G$ being a smooth transfer function [25, 30, 31, 32]. The filtering operation also might be carried out by solving PDE's [6, 7, 14, 16, 23, 24].

\footnotetext{
${ }^{*}$ Dpto. EDAN \& IMUS, Universidad de Sevilla, C/Tarfia, s/n. 41012 Sevilla, Spain \& BCAM-Basque Center for Applied Mathematics, Avda. Mazarredo, 14. 48009 Bilbao, Spain; chacon@us.es

${ }^{\dagger}$ Mathematical Research Institute of Rennes, UMR CNRS 6625, European University of Brittany \& Fluminance team, INRIA, Bat 22, Campus Beaulieu, 35042 Rennes cedex, France; Roger . Lewandowski@me.com
} 
Stresses that appear by filtering the non linear term $(\mathbf{v} \cdot \nabla) \mathbf{v}$ in the NSE, are considered to be diffusive, therefore often modeled by a turbulent diffusion term such as $-\nabla \cdot\left(\nu_{t} \nabla \overline{\mathbf{v}}\right)$, where $\nu_{t}$ is an eddy viscosity. One challenge of the LES is the determination of $\nu_{t}$.

In this paper, we study the case of a channel flow, periodic in the $x_{1}-x_{2}$ axis for simplicity. The first idea is that the projection on finite element spaces, based on a given triangulation $\left\{\mathcal{T}_{h}\right\}_{h>0}$, is a natural filtering operation, so that we seek for $\mathbf{v}_{h}$ instead of $\overline{\mathbf{v}}$, where $\mathbf{v}_{h}$ is the projection of $\mathbf{v}$ on a suitable finite element space $\mathbf{W}_{h}$. The second idea is that one can specifically model the eddy viscosity on the boundary layer by means of wall laws.

Indeed, following Kolmogorov theory [22], we consider the turbulence to be isotropic at scales small enough inside the flow domain. This assumption yields to take the eddy viscosity of a Kolmogorov-Prandtl-Smagorinsky form, $\nu_{t}=h^{2}\left|D \mathbf{v}_{h}\right|^{1}, h$ being the mixing length, which is the standard sub-grid model (SGM) [11].

However, near the wall, turbulence is not isotropic and complexity is higher than far from the wall [29], so that standard SGM cannot be used there. Usual methods proceed as follows.

First one uses the known structure of the turbulent boundary layer, as initially described by von Kármán [21] and fully developed by Schlichting [34]. Basically, the boundary layer may be split into two sub-layers, the linear sub-layer where the mean velocity profile is linear, and next, the log sub-layer where the mean velocity profile is specified by a $\log$ function. Notice that one can consider more sophisticated models to model the boundary layer [35], nevertheless always involving a log law. In all cases, those models involve an essential quantity which is the friction velocity $u_{\star}$ (see (2.4) in 2.1.ii below).

Next, one splits the domain into two subdomains, the boundary layer, and the computational domain which is the domain's part not containing the boundary layer. One then uses non linear boundary conditions at boundaries of the computational domain such as wall laws [26, 27, 28].

Based on the fact that today more computational resources are available to increase accuracy for simulating the mean flow inside the log layer, we take as computational domain the domain's part without the linear sub -layer, using an eddy viscosity of the form $\nu_{t}=h u_{\star}$ inside de log layer, deduced from standard dimensional analysis [11, 26].

To conclude the modeling process, it remains to: i) specify how $u_{\star}$ is calculated, ii) specify boundary conditions (BC) at computational domain boundaries, iii) fix the choice of the mixing length scale.

i) We assume that log law holds inside the boundary layer. Thanks to invertibility of the non linear profil, we can define $u_{\star}$ as $u_{\star}(\mathbf{v}, \mathbf{x})$, that satisfies suitable estimates (see 2.2.ii and estimate (2.17)).

ii) As the thickness of the linear sub-layer is very small compared to other scales involved in the problem, a Taylor expansion allows to deduce from the no slip condition at the flow domain boundary a Navier BC at the computational walls ( (3.6) in 3.1.i). This is as if the linear sub-layer would exert a friction over the log sub-layer.

iii) The mesh yields natural numerical length scales $h_{K}$, where $h_{K}$ is a diameter of any $K \in$ $\mathcal{T}_{h}$. Therefore, one takes $\nu_{t}$ of the form $\nu_{t}=h_{K}^{2}\left|D \mathbf{v}_{h}\right|$ on $K \in \mathcal{T}_{h}$ inside the computational domain, and $\nu_{t}=h_{K} u_{\star}(\mathbf{v}, \mathbf{x})$ on $K \in \mathcal{T}_{h}$ in the log layer (see 3.2.ii).

\footnotetext{
${ }^{1} D \mathbf{v}_{h}=(1 / 2)\left(\nabla \mathbf{v}_{h}+\nabla \mathbf{v}_{h}^{t}\right)$
} 
Once this modeling process is completed, we get a model expressed in its variational form over finite element space $\mathbf{W}_{h} \times M_{h}$, as described in 3.2.iv. So far as we know, this model is totally new, and can be generalized to more complex and realistic geometries thanks to a careful differential geometry analysis, which is a work under progress.

We consider all over the paper the steady-state case, which is in coherence with the fact that in a permanent regime and for a developed turbulence, mean fields are steady, which is not in contradiction with the fact that fluctuations might be time dependent.

We prove that this variational problem has a solution $\left(\mathbf{v}_{h}, p_{h}\right) \in \mathbf{W}_{h} \times M_{h}$ (Theorem 4.3) which converges to a solution $(\mathbf{v}, p)$ of the steady-state Navier Stokes Equation (NSE) with Navier BC (Theorem 4.4).

The paper is organized as follows. We start with general setting. Then we derive from the NSE a description of the boundary layer, introducing the friction velocity. We specify the computational domain and Navier BC, and next we perform the finite element setting and get the model. Finally we state and prove Theorem 4.3 and Theorem 4.4.

Acknowledgements: R. Lewandowski thanks Professor Li Tatsien and the ISFMA at Fudan University, Shanghaï, China, for their hospitality during spring 2012, where part of this work was performed. He also thanks E. Memin and the Fluminance team at INRIA Rennes for their support during the second term of the academic year 2012-2013. The research work of T. Chacón was partially supported by the Spanish Government Grant MTM2012-36124-C02-01.

\section{General framework}

\subsection{Channel flow}

2.1.i. Geometry, equations and boundary conditions. Let $\Omega_{f}$ be a channel periodic in the $x_{1}$ axis and $x_{2}$ axis, of height $1+2 d$ in the $x_{3}$-axis, for a small parameter $d<<1$,

$$
\Omega_{f}=\left\{\mathbf{x}=\left(x_{1}, x_{2}, x_{3}\right) \in \mathbb{T}_{2} \times \mathbb{R}^{3} \text { s. t. }-d<x_{3}<1+d\right\},
$$

where $\mathbb{T}_{2}$ is the two dimensional torus defined by

$$
\mathbb{T}_{2}=\frac{\mathbb{R}^{2}}{\mathcal{T}_{2}} \text { where } \mathcal{T}_{2}=\frac{2 \pi \mathbb{Z}^{2}}{L},
$$

and $L>0$ is a given length scale. Let $\Gamma_{f}$ denote

$$
\Gamma_{f}=\left\{\mathbf{x} \in \mathbb{T}_{2} \times \mathbb{R}^{3} \text { s. t. } x_{3}=-d \text { or } x_{3}=1+d\right\} .
$$

The steady-state Navier-Stokes equations with the no-slip boundary condition are as follows,

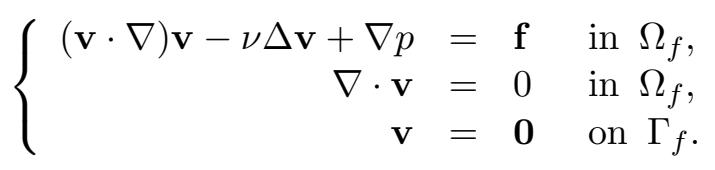

The source term $\mathbf{f}$ is a body force per mass unit, typically the gravity. Assuming $\mathbf{f} \in$ $L^{2}\left(\Omega_{f}\right)^{3}=\mathbf{L}^{2}\left(\Omega_{f}\right)$, we know that this equation has a solution $(\mathbf{v}, p) \in W^{2,3 / 2}(\Omega)^{3} \times$ $W^{1,3 / 2}(\Omega)$ (see in [36]), whose norms are bounded by constants that only depend on $\nu$, 
$\|\mathbf{f}\|_{0,2, \Omega_{f}}$ and $d$, and $p$ is defined up to a constant. Uniqueness is known when $\|\mathbf{f}\|_{0,2, \Omega_{f}} / \nu^{2}$ is small enough.

2.1.ii. Friction velocity. Let $(\mathbf{v}, p)$ be any solution of 2.3 . We still denote by $\mathbf{v}$ the trace of $\mathbf{v}$ on $\Gamma_{f}$. We deduce from trace Theorems and Sobolev Theorem that $\mathbf{v} \in W^{1,3}\left(\Gamma_{f}\right)^{3}=$ $\mathbf{W}^{1,3}\left(\Gamma_{f}\right)$. Therefore, it makes sense to consider $D \mathbf{v} \cdot \mathbf{n}$ on $\Gamma_{f}$, where $\mathbf{n}$ denotes the outward-pointing unit normal vector at $\Gamma_{f}, D \mathbf{v}=(1 / 2)\left(\nabla \mathbf{v}+\nabla \mathbf{v}^{t}\right)$. We split the vector $D \mathbf{v} \cdot \mathbf{n}$ into its tangential part and its normal part,

$$
D \mathbf{v} \cdot \mathbf{n}=(D \mathbf{v} \cdot \mathbf{n})_{\tau}+((D \mathbf{v} \cdot \mathbf{n}) \cdot \mathbf{n}) \mathbf{n} .
$$

Let $v_{\star} \in L^{6}\left(\Gamma_{f}\right)$ be defined on $\Gamma_{f}$ by

$$
v_{\star}=v_{\star}(\mathbf{v})(\mathbf{x})=\left(\nu\left|(D \mathbf{v} \cdot \mathbf{n})_{\tau}(\mathbf{x})\right|\right)^{\frac{1}{2}},
$$

called the friction velocity associated to $\mathbf{v}$ at $\mathbf{x} \in \Gamma_{f}$.

\subsection{Boundary layer description}

2.2.i. Length scale. Condition of uniqueness to system (2.3) is not satisfied in a steadystate turbulent regime. Let $\mathcal{S}$ be the set of solutions, which is a closed subset in $\mathbf{L}^{2}\left(\Omega_{f}\right)$. According to [11], one can construct a probability measure $\mu$ on $\mathcal{S}$. We consider the following velocity friction $w_{\star} \in \mathbf{L}^{6}\left(\Gamma_{f}\right)$ defined by,

$$
w_{\star}=\int_{\mathcal{S}} v_{\star}(\mathbf{v}) d \mu(\mathbf{v}) .
$$

We finally define the meanfriction velocity by

$$
u_{\star}=\frac{1}{L}\left\|w_{\star}\right\|_{0,2, \Gamma_{f}} \in \mathbb{R}
$$

to which is associated the typical length scale $\lambda$ that characterises the boundary layer,

$$
\lambda=\frac{\nu}{u_{\star}}
$$

assuming $u_{\star} \neq 0$.

We conjecture that $u_{\star} \rightarrow \infty$ when $\|\mathbf{f}\|_{0,2, \Omega_{f}} \rightarrow \infty$.

2.2.ii. Main assumption about the boundary layer structure. We focus on the bottom of $\Omega_{f},\left\{x_{3}=-d\right\}$, assuming that the boundary layer at the top $\left\{x_{3}=1+d\right\}$ has a similar structure. According to experiments (see in [34]), we assume that in the boundary layer, the mean fluid velocity has a constant direction and only depends on the variable $x_{3}$, which means $\mathbf{v}(\mathbf{x})=v\left(x_{3}\right) \mathbf{e}$, for some fixed unit vector $\mathbf{e}$. Without loss of generality, we can assume that $\mathbf{e}=\mathbf{e}_{1}$ is the unit vector pointing along the $x_{1}$-axis.

Notice that any plane $P$ of the form $P=\left\{x_{3}=h\right\}$ included in the boundary layer, and any vector $\mathbf{N}$ orthogonal at $P$ being given, our assumption yields in particular $\mathbf{v} \cdot \mathbf{N}=0$ at $P$.

2.2.iii. Log law. Experiments and suitable assumptions about turbulence [11, 34] indicate that the boundary layer can be decomposed into two sub layers:

- near the boundary where the velocity profile $v$ is linear (linear sub layer), 
- the next sub-layer specified by a log profile (log layer).

To be more specific, we introduce the dimensionless variable

$$
z^{+}=\frac{x_{3}}{\lambda}
$$

and we consider the following continuous function defined on $\left[0, z_{\text {max }}^{+}\right]$by

$$
L\left(z^{+}\right)=\left\{\begin{array}{cll}
z^{+} & \text {if } & 0 \leq z^{+} \leq z_{0}^{+} \\
\frac{1}{\kappa} \log \left(\frac{z^{+}}{z_{0}^{+}}\right)+z_{0}^{+} & \text {if } & z_{0}^{+} \leq z^{+} \leq z_{\max }^{+}
\end{array}\right.
$$

where $\kappa \approx 0,41$ is the Von Kármán constant. In practical calculations, one takes $z_{0}^{+} \approx 20$, and $z_{\max }^{+} \approx 100$, that measures the thickness of the logarithmic boundary layer, taken to be equal to $100 \lambda$. According to experiments [34], boundary layer thickness goes to zero as the Reynolds number goes to infinity.

The profile $v$ in the boundary layer at the bottom of $\Omega_{f}$ is given by the formula

$$
v\left(x_{3}\right)=u_{\star} L\left(\frac{x_{3}}{\lambda}\right) .
$$

A similar description applies to the boundary layer at the top of $\Omega_{f},\{z=1+d\}$.

2.2.iv. Friction velocity expressed as a function of the velocity. We still focus on the bottom. Any $x_{3}>0$ being given, Let

$$
F(\beta)=\beta L(\alpha \beta), \quad \alpha=\frac{x_{3}}{\nu} .
$$

With this notation, equation (2.11) may be written as

$$
v=F\left(u_{\star}\right)
$$

thanks to definition (2.8).

Lemma 2.1. Let $F:[0,+\infty) \rightarrow[0,+\infty)$ be defined by by (2.12). The function $F$ is invertible, so that equation (2.13) can be written as $u_{\star}=F^{-1}(v)$ at each given $x_{3}$.

Proof. We observe that the function $L$ satisfies

$$
\begin{aligned}
& \lim _{x \rightarrow 0^{+}} \frac{L(x)}{x}=C_{1}, \\
& \lim _{x \rightarrow \infty} \frac{L(x)}{\log x}=C_{2},
\end{aligned}
$$

where $C_{1}$ and $C_{2}$ are non-zero constants. As $L$ is strictly increasing and continuous in $(0,+\infty)$, then $F$ is strictly increasing and continuous in $(0,+\infty)$. Also, by (2.14) $F$ is continuous at $\beta=0$ with $F(0)=0$. Moreover, by (2.15) $\lim _{x \rightarrow \infty} F(x)=+\infty$. Then $F$ is bijective from $[0,+\infty)$ onto $[0,+\infty)$, which yields the invertibility of $F$ as claimed.

Lemma 2.2. Denote $h=F^{-1}$. Then there exist a constant $C=C\left(x_{3}\right)>0$, bounded, such that

$$
\forall \gamma>0, \quad h(\gamma) \leq C\left(x_{3}\right)(1+\gamma) \leq C(1+\gamma), \quad C=\sup C\left(x_{3}\right)
$$


Proof. Then $h:[0,+\infty) \mapsto[0,+\infty)$ is bijective and continuous. Also,

$$
\lim _{\gamma \rightarrow \infty} \frac{h(\gamma)}{\gamma}=\lim _{t \rightarrow \infty} \frac{t}{F(t)}=\lim _{t \rightarrow \infty} \frac{1}{L(\alpha t)}=\lim _{t \rightarrow \infty} \frac{1}{\log (\alpha t)} \frac{\log (\alpha t)}{L(\alpha t)}=0 .
$$

The conclusion is a consequence of the continuity of $h$.

We deduce from Lemma 2.1, inequality (2.16) and because top and bottom layers have the same structure, that the friction velocity can be calculated at each $\mathbf{x} \in B L$ from the velocity $\mathbf{v}$, and satisfies the estimate

$$
0<u_{\star}=u_{\star}(\mathbf{v}, \mathbf{x}) \leq C(1+|\mathbf{v}|) .
$$

\section{Turbulence model}

\subsection{Geometry and meshing}

3.1.i. Calculation domain. From now we assume that the boundary layer is included in the union of two strips,

$$
B L=\left\{-d \leq x_{3} \leq D / 2-d\right\} \cup\left\{1+d-D / 2 \leq x_{3} \leq 1+d\right\},
$$

where $d<D<<1, d$ being the order of the linear sub layer, $D$ the thikness of the global boundary layer. Standard numerical simulations are carried out in a sub domain of the flow domain that does not include the boundary layer at all, using a wall law [11, 27, 28] at artificial boundaries (walls). Our model includes the log layer, using a Navier BC based on a Taylor expansion as shown below.

The computational domain is

$$
\Omega=\left\{\mathbf{x}=\left(x_{1}, x_{2}, x_{3}\right) \in \mathbb{T}_{2} \times \mathbb{R}^{3} \text { s. t. } 0<x_{3}<1\right\},
$$

the artificial wall being defined by

$$
\Gamma_{w}=\left\{\mathbf{x} \in \mathbb{T}_{2} \times \mathbb{R}^{3} \text { s. t. } x_{3}=0 \text { or } x_{3}=1\right\} .
$$

3.1.ii. Boundary conditions. As in above, we focus on the bottom layer. By a Taylor expension we get

$$
0=\left.\left.v\right|_{x_{3}=-d} \approx v\right|_{x_{3}=0}-\left.d \frac{\partial v}{\partial x_{3}}\right|_{x_{3}=0} .
$$

From the view point of the domain $\Omega, v=\left.\mathbf{v}_{\tau}\right|_{\Gamma_{w}}$, and $\partial / \partial x_{3}=-\partial / \partial \mathbf{n}$ at $\Gamma_{w}$, where $\mathbf{v}_{\tau}$ is the tangential part of $\mathbf{v}$, defined by

$$
\mathbf{v}=\mathbf{v}_{\tau}+(\mathbf{v} \cdot \mathbf{n}) \mathbf{n},
$$

by still denoting $\mathbf{v}$ the trace of $\mathbf{v}$ at $\Gamma_{w}$, so far no risk of confusion occurs. Therefore, by remarks in 2.2.ii together with (3.4), we get

$$
\left.\mathbf{v} \cdot \mathbf{n}\right|_{\Gamma_{w}}=0,\left.\quad \frac{\partial \mathbf{v}_{\tau}}{\partial \mathbf{n}}\right|_{\Gamma_{w}}=-\frac{1}{d} \mathbf{v}_{\tau},
$$


which is a Navier boundary condition at the artificial wall, that expresses in some sense that the linear sub-layer exerts a friction on the log layer. Hence, system (2.3) becomes in $\Omega$,

$$
\left\{\begin{aligned}
(\mathbf{v} \cdot \nabla) \mathbf{v}-\nu \Delta \mathbf{v}+\nabla p & =\mathbf{f} & & \text { in } \Omega, \\
\nabla \cdot \mathbf{v} & =0 & & \text { in } \Omega, \\
\mathbf{v} \cdot \mathbf{n} & =\mathbf{0} & & \text { on } \Gamma_{w} \\
-\frac{\partial \mathbf{v}_{\tau}}{\partial \mathbf{n}} & =\frac{1}{d} \mathbf{v}_{\tau} & & \text { on } \Gamma_{w} .
\end{aligned}\right.
$$

Navier-Stokes equations with Navier boundary conditions was studied before [1, 2, 3, 10, 37], and existence of a solution to system (3.7) is already ensured.

3.1.iii. Variational formulation. Let us define the spaces

$$
\begin{gathered}
\mathbf{W}(\Omega)=\left\{\mathbf{w} \in \mathbf{H}^{1}(\Omega),\left.\quad \mathbf{w} \cdot \mathbf{n}\right|_{\Gamma_{w}}=0\right\}, \\
M(\Omega)=\left\{q \in L^{2}(\Omega), \quad \int_{\Omega_{c}} q d \mathbf{x}=0\right\},
\end{gathered}
$$

by reminding that $\mathbf{H}^{1}(\Omega)=H^{1}(\Omega)^{3}$. Strictly speaking, The space $M(\Omega)$ is isomorphic to the quotient space $L^{2}(\Omega) / \mathbb{R}$, endowed with the usual quotient norm

$$
\|\dot{p}\|_{M}=\inf _{p \in \dot{p}}\|p\|_{0,2, \Omega}
$$

It also may be viewed as a closed subspace of $L^{2}(\Omega)$ endowed with the $L^{2}(\Omega)$ norm.

The space $\mathbf{W}(\Omega)$ is endowed with the $\mathbf{H}^{1}$ norm, denoted $\|\cdot\|_{1,2, \Omega}$. As a consequence of Korn's inequality, the following usefull estimate holds,

$$
\forall \mathbf{v} \in \mathbf{W}(\Omega), \quad\|\mathbf{v}\|_{1,2, \Omega} \leq C\left(\|D \mathbf{v}\|_{0,2, \Omega}+\|\mathbf{v}\|_{0,2, \Gamma_{w}}\right),
$$

of proof of which being carried out in [10].

Let $a, b$ and $G$ the forms defined by

$$
\begin{aligned}
a(\mathbf{v}, \mathbf{w}) & =\nu(D \mathbf{v}, D \mathbf{w})_{\Omega}, \\
b(\mathbf{z} ; \mathbf{v}, \mathbf{w}) & =\frac{1}{2}\left[((\mathbf{z} \cdot \nabla) \mathbf{v}, \mathbf{w})_{\Omega}-((\mathbf{z} \cdot \nabla) \mathbf{w}, \mathbf{v})_{\Omega}\right] \\
G(\mathbf{v}, \mathbf{w}) & =\frac{\nu}{d}\left(\mathbf{v}_{\tau}, \mathbf{w}_{\tau}\right)_{\Gamma_{w}},
\end{aligned}
$$

for $\mathbf{z}, \mathbf{v}, \mathbf{w} \in \mathbf{H}^{1}(\Omega)$. Recall that when $\mathbf{z}, \mathbf{v}, \mathbf{w} \in \mathbf{W}(\Omega)$ and $\nabla \cdot \mathbf{z}=0$, then $b(\mathbf{z} ; \mathbf{v}, \mathbf{w})=$ $((\mathbf{z} \cdot \nabla) \mathbf{v}, \mathbf{w})_{\Omega}$, and $(\nabla \mathbf{z}, \nabla \mathbf{w})_{\Omega}=(D \mathbf{z}, D \mathbf{w})_{\Omega}$. Also remark that when $\mathbf{v} \in \mathbf{b}$, then $\mathbf{v}=\mathbf{v}_{\tau}$ at $\Gamma_{w}$.

We say that a pair $(\mathbf{v}, p) \in \mathbf{W}(\Omega) \times M(\Omega)$ is a weak solution of the boundary value (3.7) if it satisfies

$$
\left\{\begin{aligned}
b(\mathbf{v} ; \mathbf{v}, \mathbf{w})+a(\mathbf{v}, \mathbf{w})-(p, \nabla \cdot \mathbf{w})_{\Omega}+G(\mathbf{v}, \mathbf{w}) & =\langle\mathbf{f}, \mathbf{w}\rangle \\
(\nabla \cdot \mathbf{v}, q)_{\Omega} & =0
\end{aligned}\right.
$$

for any $(\mathbf{w}, q) \in \mathbf{W}(\Omega) \times M(\Omega)$.

3.1.iv. A priori estimate and existence result. Assume $\mathbf{f} \in \mathbf{W}(\Omega)^{\prime}$. Let $(\mathbf{v}, p)$ be any solution of Problem (3.13), and take $\mathbf{v}=\mathbf{w}$ in (3.13). From the standard formula $b(\mathbf{v} ; \mathbf{v}, \mathbf{v})=0$ that holds since $\nabla \cdot \mathbf{v}=0$ and $\mathbf{v} \cdot \mathbf{n}=0$ at $\Gamma_{w}$, we get

$$
\nu\|D \mathbf{v}\|_{0,2, \Omega}+\frac{\nu}{d}\|\mathbf{v}\|_{0,2, \Gamma_{w}}=\langle\mathbf{f}, \mathbf{v}\rangle,
$$


from where we deduce

$$
\|\mathbf{v}\|_{1,2, \Omega} \leq C \kappa^{-1} \mid \mathbf{f} \|_{\mathbf{W}(\Omega)^{\prime}}, \quad \kappa=\min \left(\nu, \frac{\nu}{d}\right)
$$

by using (3.9).

\subsection{Finite element setting}

3.2.i. Triangulation. Let $D \subset \mathbb{R}^{3}$ denotes the sample box $D=[0, L]^{2} \times[0,1]$. The computational domain $\Omega$ may be viewed as the periodic reproduction of $D$ in the $x_{1}-$ $x_{2}$ axes. Let $\left\{\mathcal{T}_{h}\right\}_{(h>0)}$ be a regular familly of triangulation of $D$, compatible with the periodicity of the domain: The restriction of the grid to the planes $x_{1}=0$ and $x_{1}=D$ is the same, and the restriction of the grid to the planes $x_{2}=0$ and $x_{2}=D$ is the same. Reproducing this triangulation by periodicity, we get a regular triangulation of $\Omega$, still denoted by $\left\{\mathcal{T}_{h}\right\}_{(h>0)}$.

In the following, for each $K \in \mathcal{T}_{h}, h_{K}=\operatorname{diam}(K)$ denotes the diameter of $K$, and

$$
h=\max _{K \in \mathcal{T}_{h}} h_{K} .
$$

3.2.ii. Eddy viscosities. We assume isotropy of the turbulence inside the domain defined by $\Omega_{\text {in }}=\Omega \backslash B L$, the boundary layer $B L$ being defined by (3.1). This yields to consider in $\Omega_{\text {in }}$ the eddy viscosity $\nu_{t, i n}$ to be of the same form as in usual Sub-Grid-Models of PrandtlKolmogorov-Smagorinsky type, where following [11], we take in each $K$ the length scale equal to $h_{K}$, leading to consider $\nu_{t, i n}$ to be of the form

$$
\nu_{t, i n}(\mathbf{v})=C_{s}^{2} \sum_{K \in \mathcal{T}_{h}} h_{K}^{2} \mathbf{1}_{K}|D \mathbf{v}|
$$

$C_{s}>0$ being an empirical constant, $\mathbf{1}_{A}$ denotes the characteristic function for any set $A$.

In the boundary layer part, $\Omega_{w}=B L \cap \Omega$, turbulence is no longer isotropic and depends on the friction velocity. Taking again $h_{K}$ as typical length scale and by a dimensional analysis argument [11], we define the eddy viscosity $\nu_{t, w}$ in $\Omega_{w}$ by

$$
\nu_{t, w}(\mathbf{v})=C_{w} \sum_{K \in \mathcal{T}_{h}} h_{K} \mathbf{1}_{K} u_{\star}(\mathbf{v}, \mathbf{x})
$$

where $C_{w}>0$ is an empirical constant and $u_{\star}$ is expressed in 2.2.iv.

Finally, the eddy viscosity we consider is of the form

$$
\nu_{t}=\nu_{t}(\mathbf{v})=\mathbf{1}_{\Omega_{i n}} \nu_{t, i n}(\mathbf{v})+\mathbf{1}_{\Omega_{w}} \nu_{t, w}(\mathbf{v})
$$

3.2.iii. Finite element spaces. The model is a mixed formulations, based upon pairs of finite element spaces $\left(\mathbf{W}_{h}, M_{h}\right) \subset \mathbf{W}(\Omega) \times M(\Omega)$, associated to the family of regular triangulations $\left\{\mathcal{T}_{h}\right\}_{h>0}$ of $\Omega$ in the sense of Ciarlet [12]. We assume that the family of pairs of spaces $\left\{\left(\mathbf{W}_{h}, M_{h}\right)\right\}_{h>0}$ satisfies the following hypothesis:

Hypothesis 1: The family of spaces $\left\{\mathbf{W}_{h} \times M_{h}\right\}_{h>0}$ is an internal approximation of $\mathbf{W}(\Omega) \times M(\Omega)$ : For all $(\mathbf{w}, p) \in \mathbf{W}(\Omega) \times M(\Omega)$ there exists a sequence $\left\{\left(\mathbf{v}_{h}, p_{h}\right)\right\}_{h>0}$ such that $\left(\mathbf{v}_{h}, p_{h}\right) \in \mathbf{W}_{h} \times M_{h}$, and

$$
\lim _{h \rightarrow 0}\left(\left\|\mathbf{v}-\mathbf{v}_{h}\right\|_{1,2, \Omega}+\left\|p-p_{h}\right\|_{0,2, \Omega}\right)=0 .
$$


Hypothesis 2: The family of pairs of spaces $\left\{\left(\mathbf{W}_{h}, M_{h}\right)\right\}_{h>0}$ satisfies the uniform discrete inf-sup condition : There exists a constant $\alpha>0$ such that

$$
\alpha\left\|q_{h}\right\|_{0,2, \Omega} \leq \sup _{\mathbf{w}_{h} \in \mathbf{W}_{h}} \frac{\left(\nabla \cdot \mathbf{w}_{h}, q_{h}\right)_{\Omega}}{\left\|\mathbf{w}_{h}\right\|_{1,2, \Omega}}, \text { for all } q_{h} \in M_{h}
$$

There is a wide literature about finite element spaces satisfying those properties (Cf. [5], [9], [15]).

3.2.iv. The model. Our LES model is expressed by the following variational problem:

Find $\left(\mathbf{v}_{h}, p_{h}\right) \in \mathbf{W}_{h} \times M_{h}$ such that for all $\left(\mathbf{w}_{h}, q_{h}\right) \in \mathbf{W}_{h} \times M_{h}$,

$$
\left\{\begin{aligned}
b\left(\mathbf{v}_{h} ; \mathbf{v}_{h}, \mathbf{w}_{h}\right)+a\left(\mathbf{v}_{h}, \mathbf{w}_{h}\right)+c\left(\mathbf{v}_{h} ; \mathbf{w}_{h}\right)+ & \\
G\left(\mathbf{v}_{h}, \mathbf{w}_{h}\right)-\left(p_{h}, \nabla \cdot \mathbf{w}_{h}\right)_{\Omega} & =\left\langle\mathbf{f}, \mathbf{w}_{h}\right\rangle, \\
\left(\nabla \cdot \mathbf{v}_{h}, q_{h}\right)_{\Omega} & =0
\end{aligned}\right.
$$

the form $c$ being defined by

$$
\begin{aligned}
& c(\mathbf{v} ; \mathbf{w})=\left(\nu_{t}(\mathbf{v}) D \mathbf{v}, D \mathbf{w}\right)_{\Omega} \text { or } \\
& c(\mathbf{v} ; \mathbf{w})=\left(\nu_{t, i n}(\mathbf{v}) D \mathbf{v}, D \mathbf{w}\right)_{\Omega_{i n}}+\left(\nu_{t, w}(\mathbf{v}) \partial_{3} \mathbf{v}, \partial_{3} \mathbf{w}\right)_{\Omega_{w}} .
\end{aligned}
$$

The second expression neglects the tangential eddy viscosity in the boundary layer, which is very small compared to the normal one.

\section{Analysis of the model}

\subsection{Technical results}

We state in this sub-section some technical results concerning the eddy viscosities and the associated turbulent diffusion form $c$, that are needed by our analysis.

4.1.i. $L^{\infty}$ eddy viscosties estimates.

Lemma 4.1. There exists a constant $C>0$ depending only on the aspect ratio of the family of triangulations $\left\{\mathcal{T}_{h}\right\}_{h>0}$ such that

$$
\left\|\nu_{t}\left(\mathbf{v}_{h}\right)\right\|_{0, \infty, \Omega} \leq C h^{1 / 2}\left\|\mathbf{v}_{h}\right\|_{1,2, \Omega} \text {, for all } \mathbf{v}_{h} \in \mathbf{W}_{h} .
$$

Proof. We start with the internal part of the eddy viscosity $\nu_{t, i n}$. Consider $\mathbf{v}_{h} \in \mathbf{W}_{h}$. As $\nabla \mathbf{v}_{h}$ is piecewise continuous, there exists $K \in \mathcal{T}_{h}$ such that

$$
\left\|\nu_{t, i n}\left(\mathbf{v}_{h}\right)\right\|_{0, \infty, \Omega}=\left\|\nu_{t, i n}\left(\mathbf{v}_{h}\right)\right\|_{0, \infty, K} \leq C_{S}^{2} h_{K}^{2}\left\|\nabla \mathbf{v}_{h}\right\|_{0, \infty, K} .
$$

By a standard finite element inverse estimate (Cf. [4]),

$$
\left\|\nabla \mathbf{v}_{h}\right\|_{0, \infty, K} \leq C h_{K}^{-3 / 2}\left\|\nabla \mathbf{v}_{h}\right\|_{0,2, K}
$$

for some constant $C>0$ depending only on the aspect ratio of the family of triangulations. Then,

$$
\left\|\nu_{t, i n}\left(\mathbf{v}_{h}\right)\right\|_{0, \infty, \Omega} \leq C C_{S}^{2} h_{K}^{2-3 / 2}\left\|\nabla \mathbf{v}_{h}\right\|_{0,2, K} \leq C C_{S}^{2} h^{1 / 2}\left\|\nabla \mathbf{v}_{h}\right\|_{0,2, \Omega},
$$


Next, we analyze the wall eddy diffusion $\nu_{t, w}$. There exists some element $K \in \mathcal{T}_{h}$ such that

$$
\left\|\nu_{t, w}\left(\mathbf{v}_{h}\right)\right\|_{0, \infty, \Omega}=\left\|\nu_{t, w}\left(\mathbf{v}_{h}\right)\right\|_{0, \infty, K} \leq C_{w} h_{K}\left(1+\left\|\mathbf{v}_{h}\right\|_{0, \infty, K}\right),
$$

where in the last inequality we have used (2.5). Using the inverse estimate (Cf. [4]), $\left\|\mathbf{v}_{h}\right\|_{0, \infty, K} \leq C h_{K}^{-1 / 2}\left\|\nabla \mathbf{v}_{h}\right\|_{0,2, K}$ we deduce

$$
\left\|\nu_{t, w}\left(\mathbf{v}_{h}\right)\right\|_{0, \infty, \Omega} \leq C^{\prime} C_{w} h^{1 / 2}\left\|\nabla \mathbf{v}_{h}\right\|_{0,2, \Omega}, \text { for some constant } C^{\prime}>0 .
$$

Combining this estimate with (4.2) and $\left\|\nabla \mathbf{v}_{h}\right\|_{0,2, \Omega} \leq\left\|\mathbf{v}_{h}\right\|_{1,2, \Omega}$, (4.1) follows.

4.1.ii. Turbulent diffusion operator properties.

Lemma 4.2. The form c defined by (3.22) satisfies the following properties:

i) $c$ is non-negative, in the sense that

$$
c(\mathbf{v} ; \mathbf{v}) \geq 0, \text { for all } \mathbf{v} \in H^{1}(\Omega)^{3} .
$$

ii) Assume that the family of triangulations $\left\{\mathcal{T}_{h}\right\}_{h>0}$ is regular. Then, for any $\mathbf{v}_{h}, \mathbf{w}_{h} \in$ $\mathbf{W}_{h}$,

$$
\left|c\left(\mathbf{v}_{h} ; \mathbf{w}_{h}\right)\right| \leq C h^{1 / 2}\left\|\mathbf{v}_{h}\right\|_{1,2, \Omega}^{2}\left\|\mathbf{w}_{h}\right\|_{1,2, \Omega},
$$

for some constant $C>0$ depending only on $d, \Omega$ and the aspect ratio of the family of triangulations.

iii) Assume that the family of triangulations $\left\{\mathcal{T}_{h}\right\}_{h>0}$ is regular. Let $\left\{\mathbf{v}_{h}\right\}_{h>0}$ and $\left\{\mathbf{w}_{h}\right\}_{h>0}$ be two sequences such that $\mathbf{v}_{h}, \mathbf{w}_{h} \in \mathbf{W}_{h}$. Then, if both sequences are bounded in $\mathbf{H}^{1}(\Omega)^{d}$,

$$
\lim _{h \rightarrow 0} c\left(\mathbf{v}_{h} ; \mathbf{w}_{h}\right)=0
$$

\section{Proof.}

i) Let $\mathbf{v} \in \mathbf{H}^{1}(\Omega)$. Then,

$$
c(\mathbf{v} ; \mathbf{v})=\int_{\Omega} \nu_{t}(\mathbf{v})|D \mathbf{v}|^{2} d \mathbf{x} \geq 0 .
$$

ii) By estimate (4.1),

$$
\begin{aligned}
\left|c\left(\mathbf{v}_{h} ; \mathbf{w}_{h}\right)\right| & \leq\left\|\nu_{t}\left(\mathbf{v}_{h}\right)\right\|_{0, \infty, \Omega}\left\|\mathbf{v}_{h}\right\|_{1,2, \Omega}\left\|\mathbf{w}_{h}\right\|_{1,2, \Omega} \\
& \leq C h^{1 / 2}\left\|\mathbf{v}_{h}\right\|_{1,2, \Omega}^{2}\left\|\mathbf{w}_{h}\right\|_{1,2, \Omega} .
\end{aligned}
$$

iii) Statement (4.4) directly follows from (4.3). 


\subsection{Existence result}

Problem (3.21) is a set of non-linear equations in finite dimension. These non-linearities are due to several effects: the convection operator, the eddy viscosity, and the wall-law boundary conditions. The space $\mathbf{W}(\Omega)$ is a closed sub-space of $\mathbf{H}^{1}(\Omega)$. Our main result is the following.

Theorem 4.3. Let $\left\{\mathcal{T}_{h}\right\}_{h>0}$ be a regular family of triangulations of the domain $\Omega$. Let $\left\{\left(\mathbf{W}_{h}, M_{h}\right)\right\}_{h>0}$ be a family of pairs of finite element spaces satisfying Hypotheses 1 and 2. Then for any $\mathbf{f} \in \mathbf{W}(\Omega)^{\prime}$ the variational problem (3.21) admits at least a solution, that satisfies the estimates

$$
\begin{aligned}
\left\|\mathbf{v}_{h}\right\|_{1,2, \Omega} & \leq C \kappa^{-1}\|\mathbf{f}\|_{\mathbf{W}(\Omega)^{\prime}}, \quad \kappa=\min \left(\nu, \frac{\nu}{d}\right) \\
\left\|p_{h}\right\|_{0,2, \Omega} & \leq C \kappa^{-1}\|\mathbf{f}\|_{\mathbf{W}(\Omega)^{\prime}}\left(\kappa^{-1} \mid \mathbf{f} \|_{\mathbf{W}(\Omega)^{\prime}}\left[1+h^{1 / 2}\right]+\nu+\frac{1}{d}+1\right) .
\end{aligned}
$$

where $C>0$ is a constant depending only on $d, \Omega$ and the aspect ratio of the family of triangulations.

Proof.. We prove the existence of solution in two steps.

4.2.i. Step 1: Existence of the velocity. Let us define the mapping $\Phi_{h}: \mathbf{W}_{h} \rightarrow \mathbf{W}_{h}^{\prime}$ as follows: Given $\mathbf{z}_{h} \in \mathbf{W}_{h}$,

$$
\left\langle\Phi_{h}\left(\mathbf{z}_{h}\right), \mathbf{w}_{h}\right\rangle=b\left(\mathbf{z}_{h} ; \mathbf{z}_{h}, \mathbf{w}_{h}\right)+a\left(\mathbf{z}_{h}, \mathbf{w}_{h}\right)+c\left(\mathbf{z}_{h} ; \mathbf{w}_{h}\right)+G\left(\mathbf{z}_{h}, \mathbf{w}_{h}\right)-\left\langle\mathbf{f}, \mathbf{w}_{h}\right\rangle,
$$

for any $\mathbf{w}_{h} \in \mathbf{W}_{h}$. This equation has a unique solution as its r.h.s. defines a linear bounded functional on $\mathbf{W}_{h}$. Moreover, the functional $\Phi_{h}$ is continuous as all functions that appear in its definition are continuous on the finite-dimensional space $\mathbf{W}_{h}$.

Consider the sub-space $Z_{h}$ of $\mathbf{W}_{h}$ defined by

$$
Z_{h}=\left\{\mathbf{w}_{h} \in \mathbf{W}_{h} \text { such that }\left(\nabla \cdot \mathbf{w}_{h}, q_{h}\right)=0, \text { for all } q_{h} \in M_{h}\right\} .
$$

$Z_{h}$ is a non-empty closed sub-space of $\mathbf{H}^{1}(\Omega)$. Then it is a Hilbert space endowed with the $\mathbf{H}^{1}(\Omega)$ norm. Let $\mathbf{z}_{h} \in Z_{h}$. Then, as $b\left(\mathbf{z}_{h} ; \mathbf{z}_{h}, \mathbf{z}_{h}\right)=0$ and $c$ is non-negative,

$$
\begin{aligned}
\left\langle\Phi_{h}\left(\mathbf{z}_{h}\right), \mathbf{z}_{h}\right\rangle & \geq a\left(\mathbf{z}_{h}, \mathbf{z}_{h}\right)+G\left(\mathbf{z}_{h}, \mathbf{z}_{h}\right)-\left\langle\mathbf{f}, \mathbf{z}_{h}\right\rangle \\
& \geq \nu\left\|D\left(\mathbf{z}_{h}\right)\right\|_{0,2, \Omega}^{2}+\frac{\nu}{d}\left\|\mathbf{z}_{h}\right\|_{0,2, \Gamma_{w}}^{2}-\|\mathbf{f}\|_{\mathbf{W}(\Omega)^{\prime}}\left\|\mathbf{z}_{h}\right\|_{1,2, \Omega} \\
& \geq \frac{C \kappa}{2}\left\|\mathbf{z}_{h}\right\|_{1,2, \Omega}^{2}-\frac{\|\mathbf{f}\|_{\mathbf{W}(\Omega)^{\prime}}^{2}}{2 C \kappa}
\end{aligned}
$$

where we have used (3.9) and Young's inequality. We deduce

$$
\forall \mathbf{z}_{h} \in Z_{h} \text { such that }\left\|\mathbf{z}_{h}\right\|_{1,2, \Omega}=\frac{\|\mathbf{f}\|_{\mathbf{W}(\Omega)^{\prime}}}{C \kappa}, \quad\left\langle\Phi_{h}\left(\mathbf{z}_{h}\right), \mathbf{z}_{h}\right\rangle_{H^{1}(\Omega)} \geq 0,
$$

Consequently, by a classical variant of Brouwer's Fixed Point Theorem (Cf. [36]), the equation

$$
b\left(\mathbf{v}_{h} ; \mathbf{v}_{h}, \mathbf{w}_{h}\right)+a\left(\mathbf{v}_{h}, \mathbf{w}_{h}\right)+c\left(\mathbf{v}_{h} ; \mathbf{w}_{h}\right)+G\left(\mathbf{v}_{h}, \mathbf{w}_{h}\right)=\left\langle\mathbf{f}, \mathbf{w}_{h}\right\rangle \quad \forall \mathbf{w}_{h} \in Z_{h}
$$

admits a solution $\mathbf{v}_{h} \in Z_{h}$ such that $\left\|\mathbf{v}_{h}\right\|_{1,2, \Omega} \leq \frac{\|\mathbf{f}\|_{\mathbf{W}(\Omega)^{\prime}}}{C \kappa}$, which precisely is (4.5) by changing $C$ in $C^{-1}$. 
4.2.ii. Step 2: Existence of the pressure. Let the operator $\mathcal{G}_{h}: M_{h} \mapsto \mathbf{W}_{h}^{\prime}$ defined by

$$
\forall q_{h} \in M_{h},\left\langle\mathcal{G}_{h}\left(q_{h}\right), \mathbf{v}_{h}\right\rangle=\left(\nabla \cdot \mathbf{v}_{h}, q_{h}\right)_{\Omega}, \text { for all } \mathbf{v}_{h} \in \mathbf{W}_{h} .
$$

Then $Z_{h}=\operatorname{Im}\left(\mathcal{G}_{h}\right)^{\perp}$. As $\operatorname{Im}\left(\mathcal{G}_{h}\right)$ is closed, then $Z_{h}^{\perp}=\operatorname{Im}\left(\mathcal{G}_{h}\right)$. As $\mathbf{v}_{h}$ is a solution of (4.8), then $\Phi_{h}\left(\mathbf{v}_{h}\right) \in Z_{h}^{\perp}$. Consequently, there exists some discrete pressure $p_{h}$ such that $\left\langle\Phi_{h}\left(\mathbf{v}_{h}\right), \mathbf{w}_{h}\right\rangle=\left(\nabla \cdot \mathbf{v}_{h}, p_{h}\right)_{\Omega}$, for all $\mathbf{w}_{h} \in \mathbf{W}_{h}$. Thus, the pair $\left(\mathbf{v}_{h}, p_{h}\right)$ solves problem (3.21). The estimate for the norm of the pressure is obtained via the discrete inf-sup condition (3.20),

$$
\left\|p_{h}\right\|_{0,2, \Omega} \leq \alpha^{-1}\left\|\Phi_{h}\right\|_{\mathbf{W}_{h}^{\prime}}
$$

for some constant $\alpha>0$. By estimates (4.3) and some standard estimates,

$$
\begin{aligned}
\left\langle\Phi_{h}\left(\mathbf{v}_{h}\right), \mathbf{w}_{h}\right\rangle & \leq C\left[\left\|\mathbf{v}_{h}\right\|_{1,2, \Omega}^{2}\left(1+C h^{1 / 2}\right)+\nu\left\|\mathbf{v}_{h}\right\|_{1,2, \Omega}\left(1+\frac{C}{d}\right)\right]\left\|\mathbf{w}_{h}\right\|_{1,2, \Omega} \\
& +\|\mathbf{f}\|_{\mathbf{W}(\Omega)^{\prime}}\left\|\mathbf{w}_{h}\right\|_{1,2, \Omega} .
\end{aligned}
$$

Then, the pressure estimate (4.6) follows from the velocity estimate (4.5).

\subsection{Convergence}

We now prove the convergence of the solution provided by method (3.21) to a weak solution of the Navier-Stokes boundary value problem model (2.3).

Theorem 4.4. Under the hypotheses of Theorem 4.3, the sequence $\left\{\left(\mathbf{v}_{h}, p_{h}\right)\right\}_{h>0}$ contains a sub-sequence strongly convergent in $\mathbf{H}^{1}(\Omega)^{2} \times L^{2}(\Omega)$ to a weak solution $(\mathbf{v}, p) \in \mathbf{W}(\Omega) \times$ $L_{0}^{2}(\Omega)$ of the steady Navier-Stokes equation (2.3). If this solution is unique, then the whole sequence converges to it.

Proof. The proof is divided into 7 steps.

4.3.i. Extracting sub sequences. By estimates (4.5) and (4.6), the sequence $\left\{\left(\mathbf{v}_{h}, p_{h}\right)\right\}_{h>0}$ is bounded in the space $\mathbf{W}(\Omega) \times L_{0}^{2}(\Omega)$ which is is a Hilbert space. Therefore, this sequence contains a subsequence, that we denote in the same way, weakly convergent in $\mathbf{W}(\Omega) \times$ $L_{0}^{2}(\Omega)$ to some pair $(\mathbf{v}, p)$. As the injection of $H^{1}(\Omega)$ in $L^{q}(\Omega)$ is compact for $1 \leq q<6$, we may assume that the subsequence is strongly convergent in $\mathbf{L}^{q}(\Omega)$ for $1 \leq q<6$, and so in particular in $\mathbf{L}^{4}(\Omega)$.

Also, the injection of $\mathbf{H}^{1 / 2}\left(\Gamma_{w}\right)$ into $L^{2}\left(\Gamma_{w}\right)$ is compact. Then we may assume that the sequence $\left\{\mathbf{v}_{h_{\Gamma_{w}}}\right\}_{h>0}$ is strongly convergent to $\mathbf{v}_{\left.\right|_{\Gamma_{w}}}$ in $\mathbf{L}^{2}\left(\Gamma_{w}\right)$.

4.3.ii. Taking the limit in the diffusion terms. Let $(\mathbf{w}, q) \in \mathbf{W}(\Omega) \times L_{0}^{2}(\Omega)$. By Hypothesis 1 , there exists a sequence $\left\{\left(\mathbf{w}_{h}, q_{h}\right)\right\}_{h>0}$ such that $\left(\mathbf{w}_{h}, q_{h}\right) \in \mathbf{W}_{h} \times M_{h}$ which is strongly convergent in $\mathbf{H}^{1}(\Omega) \times L^{2}(\Omega)$ to $(\mathbf{w}, q)$.

As $a$ is bilinear and continuous,

$$
\lim _{h \rightarrow 0} a\left(\mathbf{v}_{h}, \mathbf{w}_{h}\right)=a(\mathbf{v}, \mathbf{w}) .
$$

Next, since the sequences $\left\{\mathbf{v}_{h}\right\}_{h>0}$ and $\left\{\mathbf{w}_{h}\right\}_{h>0}$ are bounded in $\mathbf{H}^{1}(\Omega)$, we deduce from Lemma 4.2,

$$
\lim _{h \rightarrow 0} c\left(\mathbf{v}_{h} ; \mathbf{w}_{h}\right)=0 .
$$


Finally it is straightforward to check that

$$
\lim _{h \rightarrow 0} G\left(\mathbf{v}_{h} ; \mathbf{w}_{h}\right)=0 .
$$

4.3.iii. Taking the limit in limit in the convective term. We have

$$
\begin{aligned}
\left|\left(\mathbf{v}_{h} \cdot \nabla \mathbf{v}_{h}, \mathbf{w}_{h}\right)_{\Omega}-(\mathbf{v} \cdot \nabla \mathbf{v}, \mathbf{w})_{\Omega}\right| & \leq \\
\left|\left(\left(\mathbf{v}_{h}-\mathbf{v}\right) \cdot \nabla \mathbf{v}_{h}, \mathbf{w}_{h}\right)_{\Omega}\right|+\left|\left(\mathbf{v} \cdot \nabla\left(\mathbf{v}_{h}-\mathbf{v}\right), \mathbf{w}\right)_{\Omega}\right|+\left|\left(\mathbf{v} \cdot \nabla \mathbf{v}_{h}, \mathbf{w}_{h}-\mathbf{w}\right)_{\Omega}\right| & \leq \\
\left\|\mathbf{v}_{h}-\mathbf{v}\right\|_{0,4, \Omega}\left\|\nabla \mathbf{v}_{h}\right\|_{0,2, \Omega}\left\|\mathbf{w}_{h}\right\|_{0,4, \Omega} & \\
+\sum_{i, j=1}^{3}\left|\left(\partial_{j}\left(v_{h i}-v_{i}\right), v_{j} w_{i}\right)_{\Omega}\right|+\|\mathbf{v}\|_{0,4, \Omega}\left\|\nabla \mathbf{v}_{h}\right\|_{0,2, \Omega}\left\|\mathbf{w}_{h}-\mathbf{w}\right\|_{0,4, \Omega} &
\end{aligned}
$$

where we denote $\mathbf{v}_{h}=\left(v_{h 1}, v_{h 2}, v_{h 3}\right)$. All terms in the r.h.s. of the last inequality vanish in the limit because $\left\{\mathbf{v}_{h}\right\}_{h>0}$ is strongly convergent in $\mathbf{L}^{4}(\Omega),\left\{\partial_{i} v_{h i}\right\}_{h>0}$ is weakly convergent in $L^{2}(\Omega)$ and $\left\{\mathbf{w}_{h}\right\}_{h>0}$ is strongly convergent in $\mathbf{H}^{1}(\Omega)$. Then,

$$
\lim _{h \rightarrow 0}\left(\left(\mathbf{v}_{h} \cdot \nabla \mathbf{v}_{h}\right), \mathbf{w}_{h}\right)_{\Omega}=((\mathbf{v} \nabla \mathbf{v}), \mathbf{w})_{\Omega} .
$$

Similarly, $\lim _{h \rightarrow 0}\left(\left(\mathbf{v}_{h} \cdot \nabla\right) \mathbf{w}_{h}, \mathbf{v}_{h}\right)_{\Omega}=((\mathbf{v} \cdot \nabla \mathbf{w}), \mathbf{v})_{\Omega}$, and then

$$
\lim _{h \rightarrow 0} b\left(\mathbf{v}_{h} ; \mathbf{v}_{h}, \mathbf{w}_{h}\right)=b(\mathbf{v} ; \mathbf{v}, \mathbf{w}) .
$$

4.3.iv. Taking the limit in the pressure terms. Since $\left\{\nabla \cdot \mathbf{v}_{h}\right\}_{h>0}$ is weakly convergent in $L^{2}(\Omega)$ to $\nabla \cdot \mathbf{v}_{h}$ and $\left\{q_{h}\right\}_{h>0}$ is strongly convergent in $L^{2}(\Omega)$ to $q$,

$$
\lim _{h \rightarrow 0}\left(\nabla \cdot \mathbf{v}_{h}, q_{h}\right)_{\Omega}=(\nabla \cdot \mathbf{v}, q)_{\Omega}
$$

Finally, we obviously have

$$
\lim _{h \rightarrow 0}\left(p_{h}, \nabla \cdot \mathbf{w}_{h}\right)_{\Omega}=(p, \nabla \cdot \mathbf{w})_{\Omega} .
$$

Consequently, the pair $(\mathbf{v}, q)$ is a weak solution of Navier-Stokes equations (3.13).

4.3.v. Strong convergence of the velocities. Set $\mathbf{w}_{h}=\mathbf{v}_{h}$ in (3.21). Then

$$
\nu\left\|D \mathbf{v}_{h}\right\|_{0,2, \Omega}^{2}+\frac{\nu}{d}\left\|\mathbf{v}_{h}\right\|_{0,2, \Gamma_{w}}=\left\langle\mathbf{f}, \mathbf{v}_{h}\right\rangle-c\left(\mathbf{v}_{h} ; \mathbf{v}_{h}\right) .
$$

By Lemma 4.2 iii $), \lim _{h \rightarrow 0} c\left(\mathbf{v}_{h} ; \mathbf{v}_{h}\right)=0$. Therefore,

$$
\lim _{h \rightarrow 0}\left(\nu\left\|D \mathbf{v}_{h}\right\|_{0,2, \Omega}^{2}+\frac{\nu}{d}\left\|\mathbf{v}_{h}\right\|_{0,2, \Gamma_{w}}\right)=\langle\mathbf{f}, \mathbf{v}\rangle=\nu\|D \mathbf{v}\|_{0,2, \Omega}^{2}+\frac{\nu}{d}\|\mathbf{v}\|_{0,2, \Gamma_{w}}^{2},
$$

where the last equality occurs because $(\mathbf{v}, q)$ is a weak solution of Navier-Stokes equations (3.13). As $\mathbf{W}(\Omega)$ is a Hilbert space and $\left\{\mathbf{v}_{h}\right\}_{h>0}$ is weakly convergent to $\mathbf{v}$, this proves the strong convergence, since

$$
\mathbf{w} \rightarrow\left(\nu\|D \mathbf{w}\|_{0,2, \Omega}^{2}+\frac{\nu}{d}\|\mathbf{w}\|_{0,2, \Gamma_{w}}^{2}\right)^{\frac{1}{2}}
$$

is a norm equivalent to the $\mathbf{H}^{1}(\Omega)$ norm by (3.9). 
4.3.vi. Strong convergence of the pressures. We use the discrete inf-sup condition to estimate $\left\|p_{h}-p\right\|_{0,2, \Omega}$. There exists a sequence $\left\{P_{h}\right\}_{h>0}$ such that $P_{h} \in M_{h}$ for all $h>0$ which is strongly convergent in $L_{0}^{2}(\Omega)$ to $p$. We shall show that $\lim _{h \rightarrow 0}\left\|p_{h}-P_{h}\right\|_{0,2, \Omega}=0$. Let $\mathbf{w}_{h} \in \mathbf{W}_{h}$. We have

$$
\begin{aligned}
\left(p_{h}-P_{h}, \nabla \cdot \mathbf{w}_{h}\right) & =b\left(\mathbf{v}_{h} ; \mathbf{v}_{h}, \mathbf{w}_{h}\right)-b\left(\mathbf{v} ; \mathbf{v}, \mathbf{w}_{h}\right)+a\left(\mathbf{v}_{h}-\mathbf{v}, \mathbf{w}_{h}\right)+c\left(\mathbf{v}_{h} ; \mathbf{w}_{h}\right) \\
& +G\left(\mathbf{v}_{h}-\mathbf{v}, \mathbf{w}_{h}\right)+\left(p-P_{h}, \nabla \cdot \mathbf{w}_{h}\right) .
\end{aligned}
$$

As

$$
\begin{aligned}
b\left(\mathbf{v}_{h} ; \mathbf{v}_{h}, \mathbf{w}_{h}\right)-b\left(\mathbf{v} ; \mathbf{v}, \mathbf{w}_{h}\right) & =b\left(\mathbf{v}_{h} ; \mathbf{v}_{h}-\mathbf{v}, \mathbf{w}_{h}\right)+b\left(\mathbf{v}_{h}-\mathbf{v} ; \mathbf{v}, \mathbf{w}_{h}\right) \\
& \leq C\left\|\mathbf{v}_{h}-\mathbf{v}\right\|_{1,2, \Omega}\left(\left\|\mathbf{v}_{h}\right\|_{1,2, \Omega}+\|\mathbf{v}\|_{1,2, \Omega}\right),
\end{aligned}
$$

using (4.3) and the continuity of $a$ we deduce

$$
\begin{aligned}
& \left(p_{h}-P_{h}, \nabla \cdot \mathbf{w}_{h}\right) \leq C\left[\left\|\mathbf{v}_{h}-\mathbf{v}\right\|_{1,2, \Omega}\left(\left\|\mathbf{v}_{h}\right\|_{1,2, \Omega}+\|\mathbf{v}\|_{1,2, \Omega}\right)+\nu\left\|D\left(\mathbf{v}_{h}-\mathbf{v}\right)\right\|_{0,2, \Omega}\right. \\
+ & \left.h^{1 / 2}\left\|\mathbf{v}_{h}\right\|_{1,2, \Omega}^{2}+\frac{\nu}{d}\left\|\mathbf{v}_{h}-\mathbf{v}\right\|_{0,2, \Gamma_{w}}+\left\|p-P_{h}\right\|_{0,2, \Omega}\right]\left\|\mathbf{w}_{h}\right\|_{1,2, \Omega} .
\end{aligned}
$$

As $\lim _{h \rightarrow 0}\left\|\mathbf{v}_{h}-\mathbf{v}\right\|_{1,2, \Omega}=0$, then by Hypothesis $2, \lim _{h \rightarrow 0}\left\|p_{h}-P_{h}\right\|_{0,2, \Omega}=0$. Then $p_{h}$ strongly converges to $p$ in $L^{2}(\Omega)$.

4.3.vii. Uniqueness. It remains to prove that if the Navier-Stokes equations (2.3) admit a unique solution $(\mathbf{v}, p)$, then the whole sequence $\left\{\left(\mathbf{v}_{h}, p\right)\right\}_{h>0}$ converges to it. This is a standard result that holds when compactness arguments are used, which is proved by reductio ad absurdum: Assume that the whole sequence does not converge to $\left(\mathbf{v}, p_{h}\right)$. Then there exists a sub-sequence of $\left\{\left(\mathbf{v}_{h}, p_{h}\right)\right\}_{h>0}$ that lies outside some ball of $\mathbf{W}(\Omega) \times L_{0}^{2}(\Omega)$ with center $(\mathbf{v}, p)$. Then the preceding compactness argument proves that a sub-sequence of this sub-sequence would converge to the unique solution $(\mathbf{v}, p)$, what is absurd.

\section{References}

[1] Beirão Da Veiga, H.: On the regularity of flows with Ladyzhenskaya shear-dependent viscosity and slip or nonslip boundary conditions, Comm. Pure Appl. Math. 58 552577 (2005).

[2] Beirão Da Veiga, H.: Vorticity and regularity for flows under the Navier boundary condition. Commun. Pure Appl. Anal. 5 907918, (2006)

[3] Berselli, L.C.: An elementary approach to the 3D Navier-Stokes equations with Navier boundary conditions: existence and uniqueness of various classes of solutions in the flat boundary case. Discrete Contin. Dyn. Syst. Ser. S, 3 (2), 199-219, (2010)

[4] Bernardi, C., Maday, Y., Rapetti, F.: Discrétisations variationnelles de problèmes aux limites elliptiques. Mathématiques \& Applications, 45. Springer-Verlag, Berlin (2004)

[5] Bernardi C. and Raugel, G.: Analysis of some finite elements for the Stokes problem. Mathematics of Computation, 44 (169), 71-79 (1985) 
[6] Berselli, L., Lewandowski, R.: Convergence of approximate deconvolution models to the mean Navier-Stokes Equations. Annales de l'Institut Henri Poincare (C), Non Linear Analysis, 29, 171-198, (2012)

[7] Berselli, L. C., Iliescu, T., Layton, W. J.: Mathematics of Large Eddy Simulation of Turbulent Flows. Springer, Berlin (2006)

[8] Brézis, H.: Analyse fonctionelle: Théorie et applications. Masson, Paris (1983)

[9] Brezzi, F., Fortin, M.: Mixed and Hybrid Finite Element Methods. Springer-Verlag, New York (1991)

[10] Bulíček, M., Málek, J., Rajagopal, K. R,. Navier's slip and evolutionary NavierStokes-like systems with pressure and shear-rate dependent viscosity. Indiana Univ. Math. J., 56 (1), 51-85, (2007)

[11] Chacón Rebollo, T., Lewandowski, R.: Mathematical and numerical foundations of turbulence models and applications. To appear in Springer, (2013)

[12] Ciarlet, Ph.: The Finite Element Method for Elliptic Problems. Classics in Applied Mathematics, Vol. 40. SIAM, Philadelphia (2002)

[13] Clément, P.: Approximation by finite element functions using local regularization. RAIRO Anal. Numér. 9 (R-2) 77-84 (1975)

[14] Germano, M.: Differential filters for the large eddy numerical simulation of turbulent flows. Phys. Fluids 29 (6), 1755-1757 (1986)

[15] Girault, V.; Raviart, P. A. : Finite element methods for Navier-Stokes equations: theory and algorithms. Springer-Verlag (1986)

[16] Foias, C., Holm, D.D., Titi, E.S.: The Navier-Stokes-alpha model of fluid turbulence. Physica D, 152, 505-519, (2001)

[17] Hughes, T. J. R., Feijóo, G., Mazzei, L:, Quincy, J-B: The variational multiscale methoda paradigm for computational mechanics. Comp. Meth. Appl. Mech. Enrgrg. 166 (1-2), pp. 3-24 (1998).

[18] Hughes, T. J. R., Mazzei, L., Jansen, K. E.: Large eddy simulation and the variational multiscale method. Comput. Vis. Sci. 3 47-59 (2000)

[19] Hughes, T. J. R., Mazzei, L., Oberai, A., Wray, A.: The multiscale formulation of large eddy simulation. Phys. Fluids 13 (2), 505-512 (2001)

[20] Hughes, T. J. R., Oberai, Mazzei, L.: Large eddy simulation of turbulent channel flows by the variational multiscale method. Phys. Fluids 13 (6), 1784-1799 (2001)

[21] von Kármán, T. Mechanische Ähnlichkeit und Turbulenz. Nachr. Ges. Wiss. Göttingen, Math. Phys. Klasse, 58, (1930).

[22] Kolmogorov, A.N.: The local structure of turbulence in incompressible viscous fluids for very large Reynolds number. Dokl. Akad. Nauk SSR, 30, 9-13, (1941)

[23] Layton, W., Lewandowski, R.: Analysis of an eddy viscosity model for large eddy simulation of turbulent flows. Journal of Mathematical Fluid Dynamics, 4, 374-399, (2002) 
[24] Layton, W., Lewandowski, R.: A simple and stable scale similarity model for large eddy simulation: energy balance and existence of weak solutions. Applied Maths. Letters, 16, 1205-1209, (2003)

[25] Lesieur, M., Métais, O., Comte, P. Large-eddy simulations of turbulence. Cambridge University Press, (2005)

[26] Lewandowski, R.: Analyse Mathématique et Océanographie. Masson (1997)

[27] Mohammadi, M., Pironneau, O.: Analysis of the $k$-epsilon turbulence model. Masson, Paris, (1994)

[28] Parés, C.: Existence, uniqueness and regularity of solution of the equations of a turbulence model for incompressible fluids. Applicable Analysis 43, 245-296 (1992)

[29] Piomelli, U., Balaras, E.: Wall-layer models for large-eddy simulations. Annu. Fluid Mech. 34, 349-374, (2002)

[30] Pope, S.B.: Turbulent flows. Cambridge University Press, (2000)

[31] Rodi, W., Ferziger, J.H., Breuer, M., Pourquié, M.: Status of large-eddy simulation. Workshop on LES of flows past bluff bodies (Rottach-Egern, Tegernsee, 1995). ASME J. Fluid Engng., 119, 248-262, (1997)

[32] Sagaut, P.: Large Eddy Simulation for Incompressible Flows. Springer, Belin (2002)

[33] Scott, R., Zhang, S.: Finite element interpolation of non-smooth functions satisfying boundary conditions. Math. Comput. 54, 483-493 (1990)

[34] Schlichting, H. Boundary Layer Theory, 8th. Edition. Springer (2000)

[35] Spalding, D.B.: A single formula for the law of the wall. Transactions of the ASME, Series E: Journal of Applied Mechanics, 28, 455-458 (1961)

[36] Temam, R.: Navier-Stokes equations. AMS Chelsea (2001)

[37] Verfürth, R.: Finite Element approximation of incompressible Navier-Stokes equations with slip boundary conditions. Numer. Math. 50, 697-721 (1987) 